\title{
FUNCTIONAL INTESTINAL OBSTRUCTION IN THE NEWBORN
}

\author{
BY \\ ISABELLA FORSHALL, P. P. RICKHAM, and D. B. MOSSMAN \\ From the Royal Liverpool Children's Hospital
}

(Received for Publication October 5, 1950)

There are very few references in the literature to functional intestinal obstruction in the newborn. Zuelzer and Wilson in 1948 first drew attention to this condition and reported 11 cases, four of which resembled Hirschsprung's disease in older children. Vomiting and gross intestinal obstruction seen after birth were the presenting features in the remaining seven cases, five of which were operated upon and no mechanical obstruction was found; all seven died. Five were submitted to necropsy and the absence of a mechanical cause for their obstructive symptoms was confirmed; in three cases there was evidence of peritonitis. Serial sections of the intestines from three of these infants showed absence of the ganglion cells of Auerbach's plexus of the large intestine extending proximally as far as the sigmoid, caecum, and the ileum respectively. Zuelzer and Wilson were able to correlate the level of obstruction with the level of agenesis of ganglion cells. Five of the seven infants were brothers and sisters in a family of 11 children. A family with similar clinical features is reported in this paper.

\section{Case History}

Michael McG. was admitted to the Royal Liverpool Children's Hospital at the age of 4 days with the following history.

The mother's pregnancy and delivery were normal. The maternal blood was reported to be $R h$ and Kahn negative at 28 weeks of gestation and no $R h$ antibodies were present. He was considered to be a normal baby on delivery; the birth weight was $8 \mathrm{lb} .13 \mathrm{oz}$. and meconium was passed 24 hours after birth. When 48 hours old he vomited, his temperature rose to $104 \cdot 8^{\circ}$, and his respirations to 90 per minute. The following day he had well established bronchopneumonia and a grossly distended abdomen. On rectal examination the anal sphincter was rather tight and some dry meconium was present in the rectum. A plain x-ray film showed multiple small bowel fluid levels and gross intestinal distension (Fig. 1). He was transferred to the Royal Liverpool Children's Hospital as a case of intestinal obstruction.

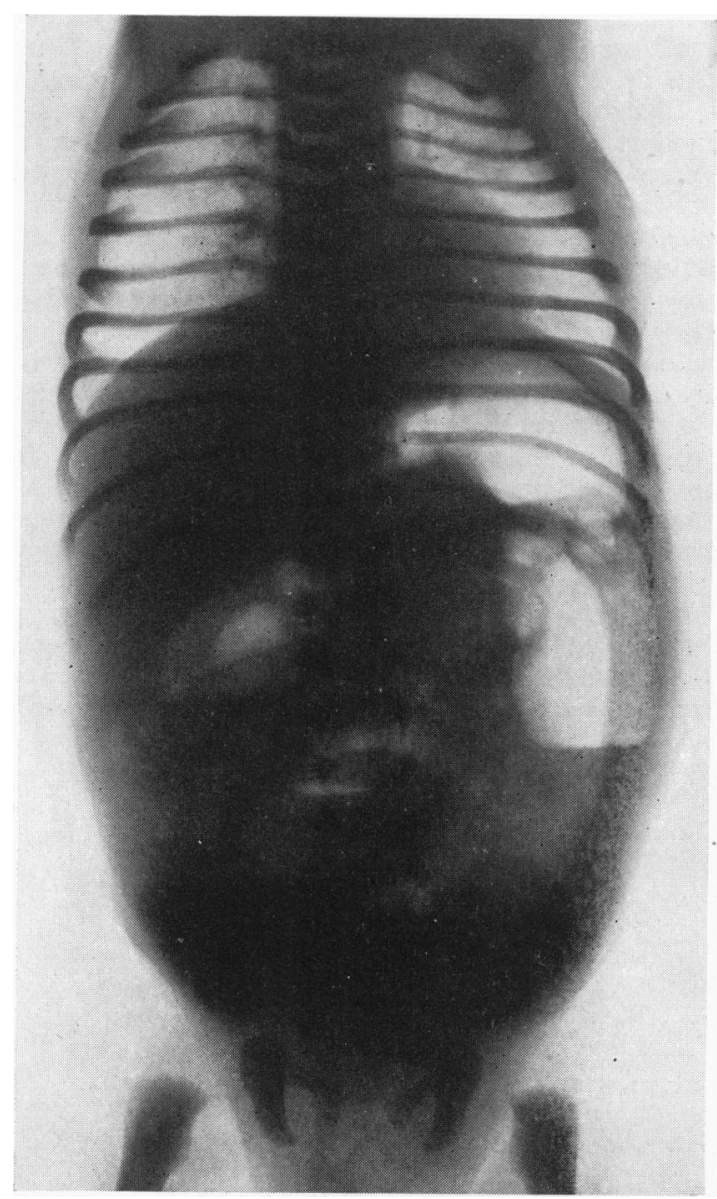

FIG. 1.-Radiograph of Michael McG. showing multiple fluid levels in the small intestine, typical of small gut obstruction.

On admission the general condition was fair; there was very marked distension of the abdomen and two enemata gave no result. Gastric suction and intravenous infusion were instituted. Pancreatic fibrosis as 
a possible diagnosis was considered unlikely because trypsin was present in the duodenal juice.

Six hours after admission a laparotomy was performed under endo-tracheal ether, oxygen, and $\mathrm{CO}_{2}$ anaesthesia (Dr. Jackson Rees). The abdomen was opened through a left paramedian incision. The lower jejunum and whole ileum were grossly distended, the caecum and ascending colon moderately so, but the transverse and descending colon were normal in calibre. There was no clear line of demarcation between the distended and normal bowel and no mechanical obstruction. The distended bowel was dusky blue. There was some enlargement of the mesenteric glands but no obstruction to the mesenteric vessels. No other abnormality was noted and the abdomen was closed.

Gastric suction and intravenous fluids were continued post-operatively and the child was nursed in an oxygen tent. Abdominal distension was marked. On the fourth post-operative day he passed a small stool and some flatus, and was given small feeds by mouth. No further stools were passed and the distension recurred in spite of enemata.

On the thirteenth post-operative day he had a violent attack of vomiting and ruptured his abdominal incision. The wound was re-sutured under general anaesthesia and gastric suction and intravenous fluids were reinstituted.

Six days after the second anaesthetic he took feeds well and vomited only occasionally. He was, however, extremely constipated, although enemata produced small stools and some flatus. There were two further episodes of distension and temporary improvement. At 5 weeks of age the vomiting started again, enemata failed to relieve the distension, and the child died.

Post-mortem Findings. The body weighed $8 \mathrm{lb} .2 \mathrm{oz}$. There was marked abdominal distension with wasting of the limbs. Peritonitis was present with numerous abscess cavities, and the coils of intestine were matted together by a thick fibro-purulent exudate. On the anterior aspect of the caecum there was a perforated stercoral ulcer, $1 \mathrm{~cm}$. in diameter. The terminal ileum, caecum, and proximal half of the ascending colon were grossly dilated and thin walled, but the rest of the large bowel was of normal calibre. The whole intestine contained hard, compact, homogeneous, malodorous faeces.

Nothing of significance was found in the remaining viscera including the pancreas.

Microscopical Findings. Blocks, both transverse and longitudinal, were taken from the small bowel, vermiform appendix, descending and pelvic colon, and rectum. The most distal blocks came from within a few centimetres of the anal sphincter, but the sphincter ani itself was not included.

From each block four to six sections were cut and stained with haematoxylin and eosin and several frozen sections were stained. Myenteric and sub-mucous ganglia were present in the small bowel and in the ileocaecal region in normal numbers, but in the caecum the myenteric ganglia became scanty, one or two being found in each section of some $2 \mathrm{~cm}$. in length. Normal ganglia consist of a dozen or more neurons, but these ganglia

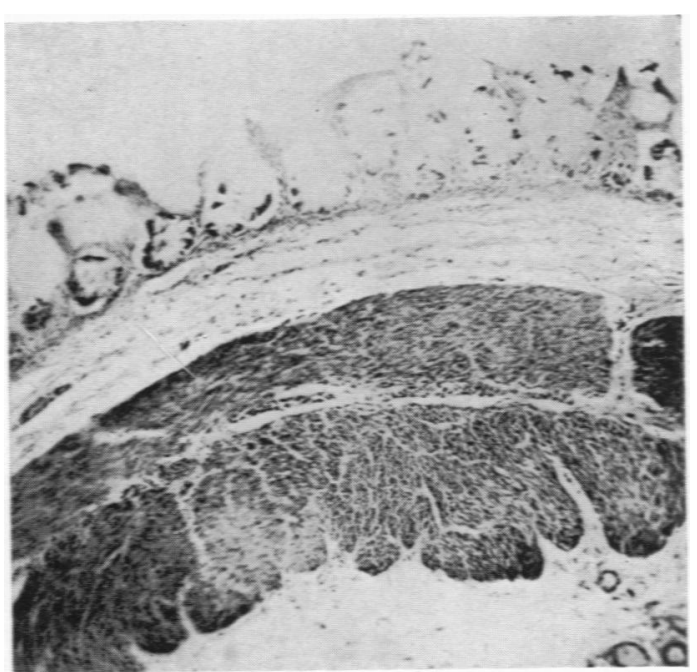

Fig. 2.-Normal colon. Myenteric ganglia can be seen separating the muscle coats. $\times 56$.

showed only two to four neurons. The sparseness of ganglia could in part be explained by the dilatation found at this site and consequent dilution by stretching. The appendix was so inflamed that the presence or absence of ganglia could not be determined.

As the caecum merged into the ascending colon ganglia became progressively more difficult to find, and at about the middle third of the ascending colon, examination of several sections failed to reveal any ganglia. From this point onwards to the last rectal section not a single myenteric ganglion was found. It was thought that the submucous ganglia were absent from the same regions,

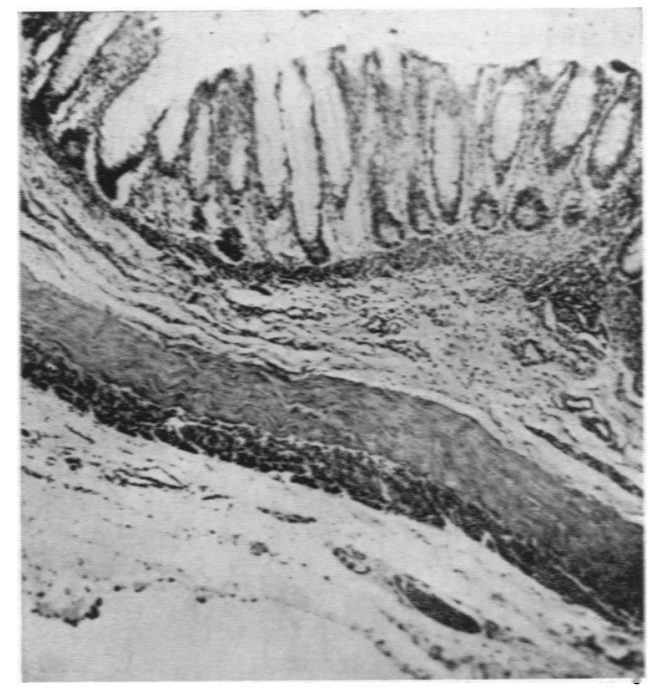

Fig. 3.-Colon of Michael McG. showing closely approximated muscle coats. No ganglia. $\times 56$. 


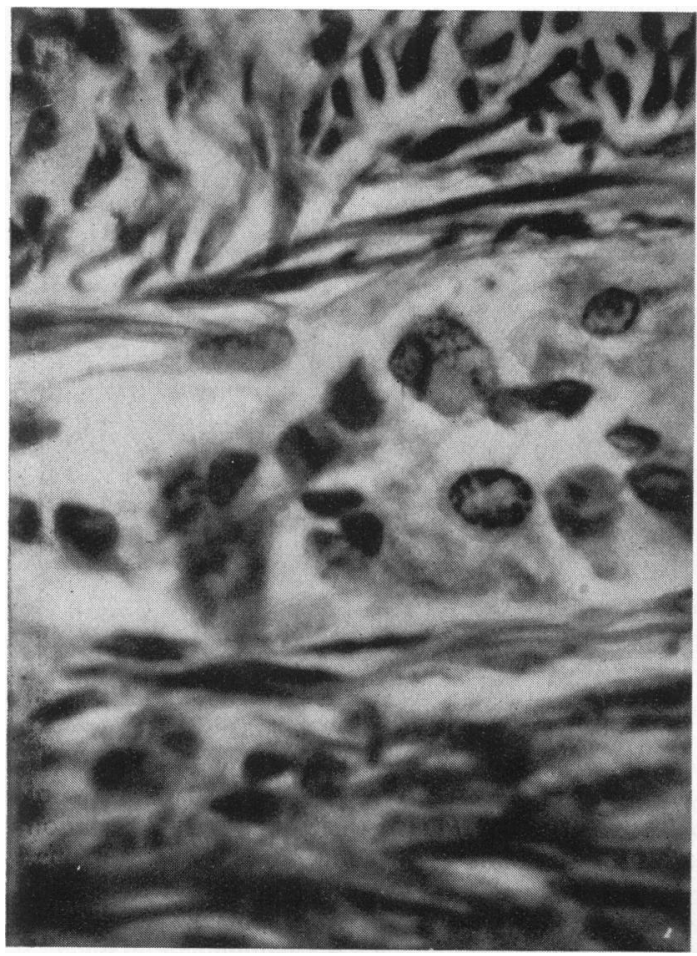

FIG. 4.-Normal myenteric ganglion. $\times 800$.

but the masking effect of considerable numbers of inflammatory cells throughout the submucosa of the greater part of the large bowel made it difficult to establish this impression.

At the hepatic flexure small, non-myelinated nerve bundles, well circumscribed by a delicate connective tissue sheath, were seen coursing between the longitudinal and circular muscle coats. The general direction of these bundles appeared to be longitudinal as they were cut more often at right angles in transverse than in longitudinal sections (Fig. 5). They remained scanty in the proximal colon; distally they increased in size and number becoming both numerous and conspicuous in the pelvic colon and rectum. In the caecum, ascending and transverse colon, the longitudinal and circular muscle coats were closely approximated owing to the absence of myenteric ganglia (Fig. 3). In the descending colon and rectum the normal separation was maintained by the non-myelinated nerve bundles.

The accompanying photomicrographs illustrate the conditions found in the pathological gut. Photographs of normal sections are included for comparison (Figs. 2 and 4).

Family History. Michael McG's family tree (see page 300) shows that three of his four brothers and sisters died shortly after birth; two were operated upon at the Royal Liverpool Children's Hospital. One of Michael's maternal aunts has for five years been under treatment for her abdominal condition at the same hospital. The brief case histories of these children are as follows:

(1) Stephen McG. was born on March 30, 1942. The pregnancy and delivery were normal. The birth weight was $7 \mathrm{lb}$. $11 \mathrm{oz}$. He died 48 hours after birth. The report of the post-mortem examination, performed at another hospital, reads: 'Congestion of lungs and intestines.' No sections of the intestines were examined microscopically.

(2) Patricia McG. was born on May 19, 1947, following a normal pregnancy and delivery. She was admitted to the Royal Liverpool Children's Hospital at the age of 8 weeks with a history of constipation since birth. At 4 weeks of age vomiting began, and this was temporarily relieved when bottle feeds were given instead of breast feeds, but two days before admission vomiting had started again. On examination the abdomen was seen to be grossly distended and the rectum was full of faeces. An enema yielded a small constipated result. The following day distension was more marked and gastric suction and intravenous infusion were started. At laparotomy there was dilatation of small and large intestine with some pus in the right iliac fossa. The appendix was not found. The abdomen was closed with drainage. Post-operatively the belly remained distended and gastric suction and intravenous infusion were continued. She improved and took feeds quite

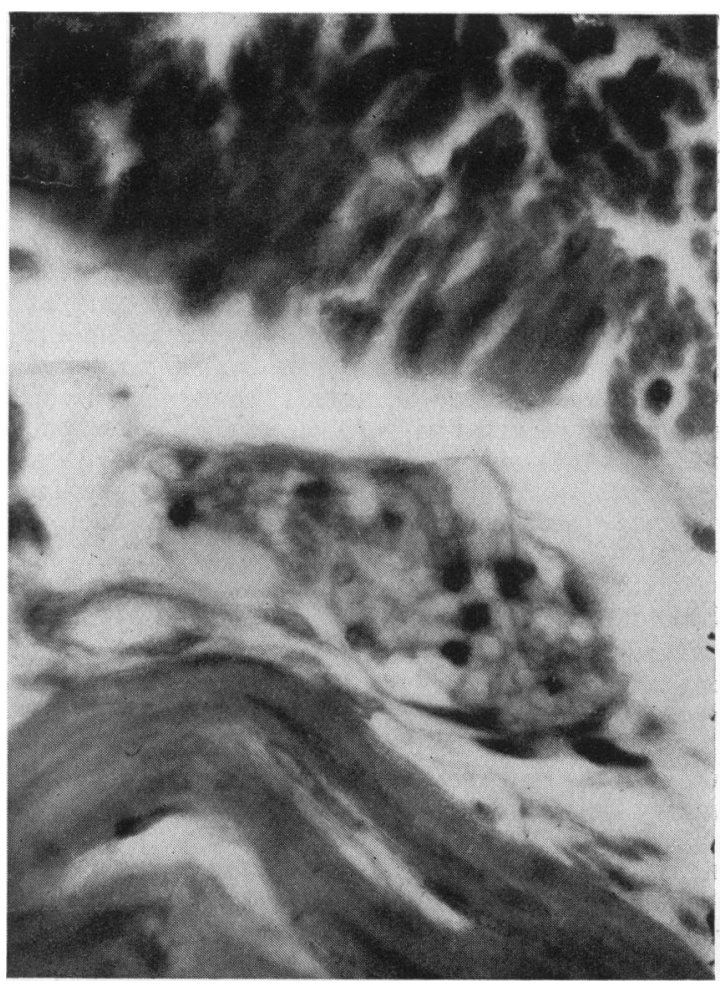

Fig. 5.-Plexus from Michael McG. Ganglionic neurons absent. $\times 800$. 
well but remained very constipated. Four weeks later her condition deteriorated, there was gross distension, and she died at the age of 14 weeks. Post-mortem examination revealed multiple adhesions and pockets of pus in the abdomen. There was no mechanical obstruction. No sections were taken from the intestine for microscopical examination.

(3) Anthony McG. was born on October 10, 1948, after a normal pregnancy and delivery. His birth weight was $7 \mathrm{lb} .11 \mathrm{oz}$. He was admitted to the Royal Liverpool Children's Hospital when one day old with a history of vomiting bile. The abdomen was distended and an enema yielded a small amount of meconium. Intravenous saline and gastric suction were started. At laparotomy distended intestine was found which ended abruptly with undistended gut ${ }^{\circ}$ following on. There was no mechanical obstruction. Post-operatively, because of distension, gastric suction and intravenous infusion were given. Four days later his condition had improved and he took feeds well. During the next few days he passed frequent, small, yellow, watery stools but then again became constipated. At 18 days the abdomen became very distended and he started to vomit copiously; an enema yielded some flatus only. He died at the age of 23 days. During the last three weeks of life he had daily enemata and stomach wash-outs, and was given ' amechol,' $\frac{1}{8}$ of a tablet, daily. Permission for post-mortem examination was not granted.

(4) Kathleen O'C. was born on September 2, 1942, following a normal pregnancy and delivery. She attended the Royal Liverpool Children's Hospital at the age of 2 years with a history of attacks of vomiting for three months. During the next six months she was admitted to hospital on several occasions with bouts of vomiting and abdominal distension, necessitating gastric suction and intravenous infusion. She was not seen again until she was 6 years old when she returned to the outpatient department with a history that for the last four years she had suffered from attacks of copious vomiting of green fluid every four or six weeks. Her bowels opened regularly. On examination the only abnormal finding was abdominal distension. All pathological investigations of blood, urine, stools, and duodenal juices were negative. A barium follow-through showed an enormously dilated coil of jejunum (Figs 6 and 7). For the next three months she was again in and out of hospital with attacks of distension and vomiting necessitating gastric suction and intravenous infusions. Several barium meals were given and always showed the same picture. At laparotomy the duodenum and first part of the jejunum were found to be enormously dilated, and the dilated intestine gradually tapered off into normal

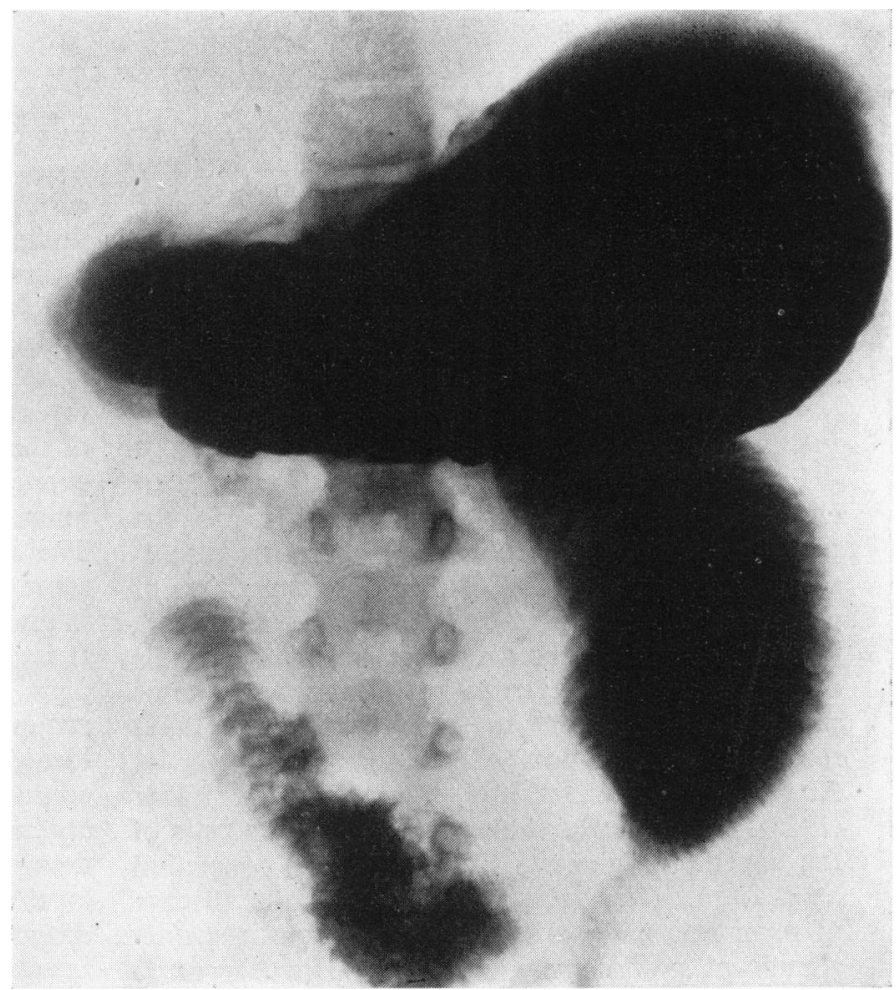

Fig. 6.-Radiograph of Kathleen O'C. one hour after barium meal.

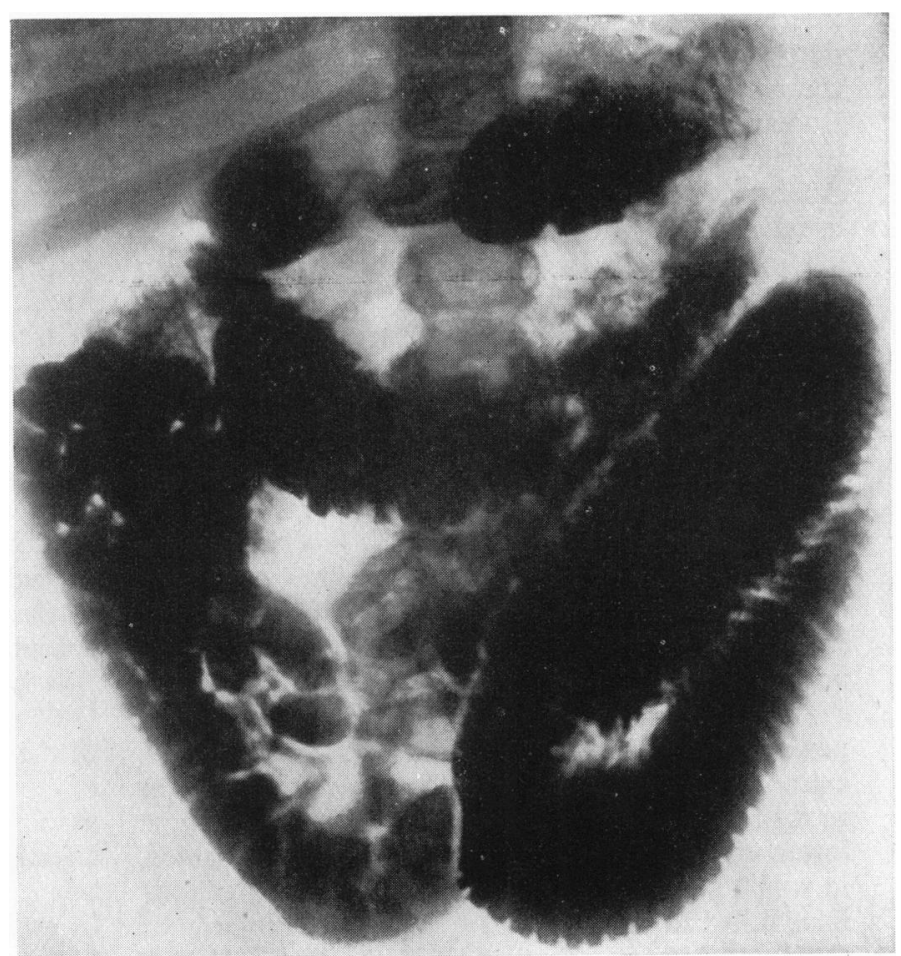

FIG. 7.-Radiograph of Kathleen O'C. three hours after barium meal. 
bowel. There was no mechanical obstruction. From the date of operation there has been gradual improvement. The attacks of vomiting have become less forceful and frequent. Her bowels open regularly, but she still has a distended abdomen.

Very little is known about the family history of grandmother $\mathrm{O}^{\prime} \mathrm{C}$., but it will be noted from the family tree that some of her brothers and sisters and some of her nephews and nieces, died of ' fits' in infancy.

\section{Discussion}

Ever since Hirschsprung in 1888 described the clinical syndrome which bears his name, the pathology of this condition has been under discussion. As early as 1901, Tittel noticed scantiness of ganglion cells in the myenteric plexus. Cameron, in 1928, discussing the aetiology of Hirschsprung's disease, described degeneration of ganglia in some sections, and Robertson and Kernohans (1938) recorded degeneration of the ganglion cells of Auerbach's plexus. Tiffin, Chandler, and Faber (1940), found localized absence of ganglion cells of the myenteric plexus in one case of congenital megacolon. The delay in recognizing the absence of myenteric ganglia as the major, if not the only significant pathological change in Hirschsprung's disease, was undoubtedly due to failure to differentiate clearly between cases of Hirschsprung's disease and other types of megacolon. Renewed interest in Hirschsprung's disease and its pathology date from 1948 when Swenson and Bill published the result of cases treated by recto-sigmoidectomy. Bodian, Stephens, and Ward (1949) have shown that in true Hirschsprung's disease the ganglion cells of Auerbach's plexus are absent from the rectum and usually from the lower sigmoid also.

Hirschsprung's disease is known to have an occasional familial basis; it is also occasionally diagnosed at birth or soon afterwards. Bodian et al. (1949) record three cases in the neonatal period and Perrot and Danon (1935) record another neonatal case. In these cases, however, the disease affected the rectum and sigmoid only and they were therefore capable of cure by recto-sigmoidectomy. Swenson (personal communication) has had several survivals following operation in the newborn. The cases reported here, however, differ from Hirschsprung's disease in the severity of the symptoms and the invariably fatal outcome.

Interest in functional intestinal obstruction of the newborn was awakened by the McG. family with its extraordinary familial incidence. However, in retrospect we believe that other cases presenting as lower small bowel obstruction in newborn babies, in which laparotomy and radiological investigations have failed to reveal a cause, have probably been of the same nature. Further, it seems probable that this condition is not uncommon. These cases are likely to remain unpublished, as surgeons are obviously reluctant to publish cases where the findings at laparotomy are so unsatisfactory. Necropsy also will be uninformative unless microscopic examination by serial sections of the gut is undertaken.

Aetiology. We have shown that in our case the myenteric ganglia were absent from the greater part of the large bowel. It is now well known that these changes are observed in the rectum or recto-sigmoid junction in cases of classical Hirschsprung's disease. The case here described thus appears to have the same aetiology as Hirschsprung's disease but differs in that a much greater segment of bowel was affected. It is current teaching that preganglionic vagal and parasympathetic nerve fibres end as arborizations around neurons of the myenteric plexus and that local intestinal reflex movements are initiated and controlled through neurons in this plexus. In the absence of these nerve cells the affected segment is deprived of both parasympathetic innervation and local reflexes, with resultant absence of peristalsis. Attempts by the normally innervated proximal intestine to push the bowel contents past the inert segment appear to be responsible for the muscular hypertrophy usually observed in Hirschsprung's disease. No such hypertrophy was observed in our case, presumably because the child did not live long enough. Zuelzer and Wilson (1948) have demonstrated that hypertrophy is roughly proportional to the time of survival.

Cameron (1928) suggested that the absence of the myenteric ganglia could be attributed to inflammation. We suggest that the occasional familial incidence of this condition, as exemplified in the McG. family and the family quoted by Zuelzer and Wilson (1948), favours a congenital abnormality of the neuro-muscular mechanism, probably a neural agenesis.

A point of interest is the presence of the ganglia in approximately the area supplied by the vagus and their absence from that supplied by the sacral outflow. We were, however, not able to determine the exact distribution of the vagus and sacral nerves to the large bowel and the matter therefore remains speculative.

It is difficult to resist the inference that Kathleen $O ' C$. suffered from the same condition in a segment of the jejunum. If she suffered from the same type of condition at a higher level, then it must be assumed that in a proportion of these cases the affected segment will not include the most distal bowel. Such cases would be amenable to treatment by excision or short-circuiting operations. Swenson (personal communication) has had a patient with 
segmental affection of the ileum, cured by excision of the segment. On section the excised part of the ileum showed absence of ganglion cells of the myenteric plexus.

Although Kathleen had recurrent attacks of vomiting, obstruction never became absolute and the child survived. It seems possible that the difference in the site of the lesion explains the difference in prognosis. The area of inert bowel may possibly not present the same resistance to fluid as to solid intestinal contents.

We believe it to be the first time that this clinical syndrome has been described in at least two successive generations. We should add that intestinal radiographs of Michael McG's mother were normal.

Treatment. It seems probable that in the future these children will be submitted to laparatomy. At the time of operation a rough estimate of the level of agenesis of ganglion cells can be made by observing the level of dilated and non-dilated bowel. In cases where the descending colon only is affected, a transverse colostomy appears to be justifiable. In cases of involvement proximal to the middle of the transverse colon, an ileostomy or caecostomy is feasible. Babies stand all types of enterostomy badly; even transverse colostomy may be poorly tolerated. When we operated upon Michael McG. we believed that an ileostomy would lead to rapid death from dehydration, but we have since heard that Swenson (personal communication) has in fact been able to perform a successful terminal ileostomy in one of these cases.

\section{Summary}

Functional intestinal obstruction of the newborn affecting several members of one family is described. The possible relationship of this condition to Hirschsprung's disease and the treatment are discussed. We suggest that functional intestinal obstruction in the newborn, with or without a familial basis, is a definite clinical and pathological entity.

We should like to thank Professor Norman B. Capon for referring Michael and Anthony McG. and Kathleen O'C. to us, and Mr. Philip H. Hawe for permission to record the case of Patricia McG. Our thanks are also due to Miss J. Henderson who prepared the histological material.

\section{REFERENCES.}

Bodian, M., Stephens, F. D., and Ward, B. C. H. (1949). Lancet, 1, 6.

Cameron, J. A. M. (1928). Archives of Disease in Childhood, 3, 210.

Hirschsprung, H. (1888). Jb. Kinderheilk., 27, 1.

Perrot, A., and Danon, L. (1935). Ann. Anat. path., $12,157$.

Robertson, H. E., and Kernohan, J. W. (1938). Proc. Mayo Clin., 13, 123.

Swenson, O., and Bill, A. H. (1948). Surgery, 24, 212.

Tiffin, M. E., Chandler, L. R., and Faber, H. K. (1940). Amer. J. Dis. Child., 59, 1071.

Tittel, K. (1901). Wien. klin. Wschr., 14, 903.

Zuelzer, W. W., and Wilson, J. L. (1948). Amer. J. Dis. Child., 75, 40. 


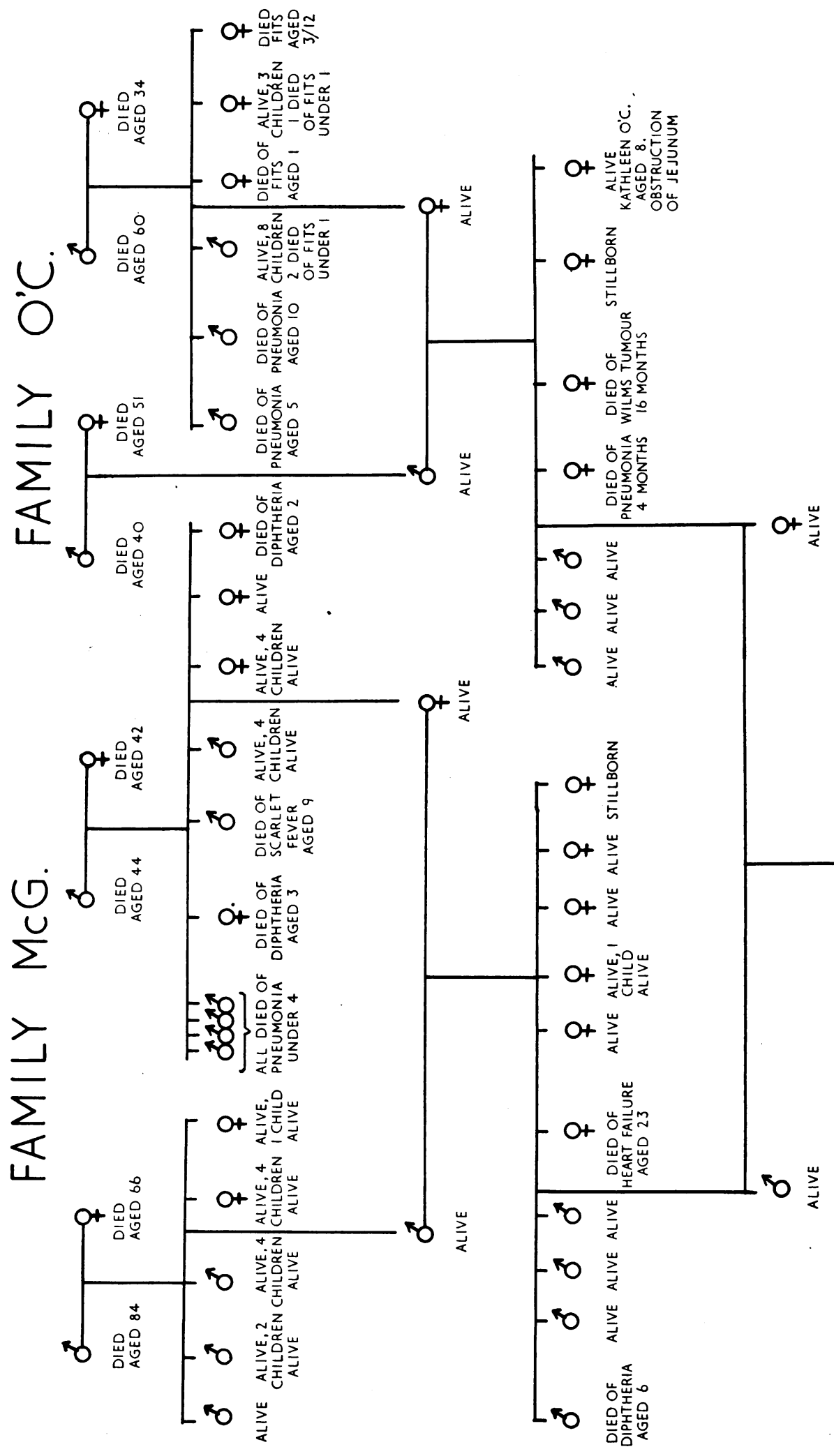

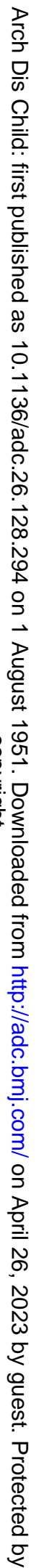

